\title{
Full separability criterion for tripartite quantum systems
}

\author{
Chang-shui $\mathrm{Yu}$ and He-shan Song* \\ Department of Physics, Dalian University of Technology, \\ Dalian 116024, China
}

(Dated: May 3, 2019)

\begin{abstract}
In this paper, an intuitive approach is employed to generalize the full separability criterion of tripartite quantum states of qubits to the higher-dimensional systems (Phys. Rev. A 72, 022333 (2005)). A distinct characteristic of the present generalization is that less restrictive conditions are needed to characterize the properties of full separability. Furthermore, the formulation for pure states can be conveniently extended to the case of mixed states by utilizing the kronecker product approximate technique. As applications, we give the analytic approximation of the criterion for weakly mixed tripartite quantum states and investigate the full separability of some weakly mixed states.
\end{abstract}

PACS numbers: 03.67.Mn, 03.65.Ud, 42.50.Ct

\section{INTRODUCTION}

Entanglement, as an essential ingredient of quantum information theory, has been an important physical resource for a lot of quantum protocols, such as quantum computation [1], quantum cryptography [2], quantum teleportation [3], quantum dense coding [4] and so on. Recently, many efforts have been made to characterize the quantatively properties of entanglement [5-8], however, the good understanding is only restricted to lowdimensional systems. The quantification of entanglement for higher dimensional systems and multipartite quantum systems remains an open question.

Since Coffman et al. [9] introduced the so called residual entanglement on the basis of concurrence [5], the investigation of multipartite entanglement has attracted much attention. For example, Dür et al. have considered the classification of entanglement for tripartite systems of qubits [10]; Miyake [11] has given the classification for multipartite systems based on the hyperdeterminant. On the basis of the different classes of multipartite entanglement, the corresponding entanglement monotones can be given $[11,12]$. Some quantities have also presented to characterize the properties of entanglement by collecting the contributions of the entanglements of different classes $[13,14]$. One can note that the quantities introduced in Refs. $[13,14]$ can also characterize the full separability of a pure multipartite state. However it is easily found that construction of these quantities requires more restrictive conditions. Even though some conditions may be repeated, it is usually not easy to exclude the repeated ones, especially for high-dimensional systems. Hence, it will reduce the efficiency of calculation to some extent.

Considering the full separability criterion introduced in Ref. [15], which can effectively reduce the restrictive conditions to some extent, in this paper, we will gen-

*Electronic address: hssong@dlut.edu.cn eralize the criterion to high-dimensional systems by an intuitive approach. The generalized full separability criterion for pure states can be conveniently extended to the case of mixed states by utilizing the kronecker product approximate technique which can usually further reduce restrictive conditions. As applications, we give the analytic approximation of the criterion for weakly mixed tripartite quantum states and study the full separability of some weakly mixed states. The paper is organized as follows. Firstly, we give the intuitive generalization of the separability criterion for pure states; secondly, we extend it to mixed states and discuss the full separability of some quasi pure states; the conclusions are drawn in the end.

\section{FULL SEPARABILITY CRITERION FOR TRIPARTITE PURE STATES}

At first, let us recall the full separability criterion for tripartite pure states of qubits given in Ref. [15]. A tripartite pure state $|\psi\rangle_{A B C}$ denoted by a vector in $2 \times$ $2 \times 2$ dimensional Hilbert space,

$$
|\psi\rangle=\left(a_{000}, a_{001}, a_{010}, a_{011}, a_{100}, a_{101}, a_{110}, a_{111}\right)^{T}
$$

with the superscript $T$ denoting transpose, is fully separable, if and only if

$$
C(|\psi\rangle)=\mid \boldsymbol{C}(|\psi\rangle) \mid=\sqrt{\sum_{\alpha}\left|C^{\alpha}\right|^{2}}=0,
$$

here the vector $\boldsymbol{C}(\psi)=\stackrel{9}{\oplus} C_{\alpha=1}^{\alpha}$ with $C^{\alpha}=\left\langle\psi^{*}\left|s^{\alpha}\right| \psi\right\rangle$, where the star denotes complex conjugation, and

$$
s^{1}=-\sigma_{y} \otimes \sigma_{y} \otimes I_{1}, s^{2}=-\sigma_{y} \otimes \sigma_{y} \otimes I_{2}, s^{3}=-\sigma_{y} \otimes I_{1} \otimes \sigma_{y},
$$

$s^{4}=-\sigma_{y} \otimes I_{2} \otimes \sigma_{y}, s^{5}=-I_{1} \otimes \sigma_{y} \otimes \sigma_{y}, s^{6}=-I_{2} \otimes \sigma_{y} \otimes \sigma_{y}$, 
$s^{7}=-\sigma_{x} \otimes \sigma_{y} \otimes \sigma_{y}, s^{8}=-\sigma_{y} \otimes \sigma_{x} \otimes \sigma_{y}, s^{9}=-\sigma_{y} \otimes \sigma_{y} \otimes \sigma_{x}$,

with $\sigma_{x}=\left(\begin{array}{ll}0 & 1 \\ 1 & 0\end{array}\right), \sigma_{y}=\left(\begin{array}{cc}0 & -i \\ i & 0\end{array}\right), I_{1}=\left(\begin{array}{ll}1 & 0 \\ 0 & 0\end{array}\right)$ and $I_{2}=\left(\begin{array}{ll}0 & 0 \\ 0 & 1\end{array}\right)$.

As mentioned in Ref. [15], a tripartite pure state of qubits can be considered as a tensor cubes. Directly, a tripartite higher-dimensional pure state can naturally considered as a tensor grid which includes tensor cubes. E.g. let $\left|\phi_{A B C}\right\rangle=\sum_{i, j=0}^{1} \sum_{k=0}^{2} a_{i j k}|i j k\rangle_{A B C}$, the tensor grid of $\left|\phi_{A B C}\right\rangle$ can be pictured as two adjoining cubes, which includes three tensor cubes. In this sense, one can draw a conclusion that tensor cube can be regarded as the unit of tensor grid. Since every tensor cube in a tensor grid can be considered as an non-normalized tripartite pure state of qubits, one can get that every unit corresponds to a $C$ defined in eq. (1). Therefore, the tensor cube can also be considered as a unit which describes the full separability of a tripartite higher-dimensional pure state. In other words, the full separability of the given tripartite higher-dimensional pure state can be described by the full separability of the non-normalized tripartite pure state of qubits.

Theorem 1:-For any a tripartite pure state $|\chi\rangle$ which includes $M$ non-normalized tripartite pure states of qubits (tensor cubes mentioned above), let the nonnormalized pure state of qubits corresponding to the $i$ th cube be denoted by $\left|\varphi_{i}\right\rangle$, one can obtain the corresponding $C\left(\left|\varphi_{i}\right\rangle\right)$. Define

$$
\mathcal{C}(|\chi\rangle)=\sqrt{\sum_{i=1}^{M} C^{2}\left(\left|\varphi_{i}\right\rangle\right)}
$$

for the state $|\chi\rangle$, then $|\chi\rangle$ is fully separable, if and only if $F(|\chi\rangle)=0$.

Proof. It is obvious that $\mathcal{C}(|\chi\rangle)=0$ means that $C\left(\left|\varphi_{i}\right\rangle\right)=0$ holds for all $\varphi_{i}$, vice versa. Since the tensor cube corresponds to the unit of describing full separability, $\mathcal{C}(|\chi\rangle)=0$ shows that there does not exist any entanglement in $|\chi\rangle$. That is to say, the tripartite quantum state $|\chi\rangle$ is fully separable. In other words, since every non-normalized $\varphi_{i}$ is fully separable, one can obtain that every group of parallel lines of the tensor grid is linear dependent. I.e. the state that the grid denotes is fully separable [15]. On the contrary, if $|\chi\rangle$ is fully separable, $C\left(\left|\varphi_{i}\right\rangle\right)=0$, i.e. $\mathcal{C}(|\chi\rangle)=0$.

Considering the matrix notation of

$$
|\chi\rangle=\sum_{i=0}^{n_{1}-1} \sum_{j=0}^{n_{2}-1} \sum_{k=0}^{n_{3}-1} a_{i j k}|i j k\rangle
$$

$\mathcal{C}(|\chi\rangle)$ can be expressed as the function of $|\chi\rangle$, i.e.

$$
\mathcal{C}(|\chi\rangle)=\sqrt{\sum_{\alpha=1}^{N_{1}} \sum_{\beta=1}^{N_{2}} \sum_{\gamma=1}^{N_{3}} C^{2}\left(\left(s_{\alpha} \otimes s_{\beta} \otimes s_{\gamma}\right)|\chi\rangle\right)}
$$

where $N_{p}=\frac{n_{p}\left(n_{p}-1\right)}{2}$ with $p=1,2,3 ; s_{q}, q=\alpha, \beta, \gamma$, denotes $2 \times n_{p}$ matrix with $p$ corresponding to $q$. If the generator of the group $S O\left(n_{p}\right)$ is denoted by $S_{p}, s_{q}$ can be derived from $\left|S_{p}\right|$ by deleting the row where all the elements are zero, where || denotes the absolute value of the matrix elements.

According to eq. (1), eq. (6) can be expanded by

$$
\begin{aligned}
\mathcal{C}(|\chi\rangle)= & \sqrt{\sum_{\alpha=1}^{N_{1}} \sum_{\beta=1}^{N_{2}} \sum_{\gamma=1}^{N_{3}} \sum_{\delta=1}^{9} \mid\left. C^{\delta}\left(\left(s_{\alpha} \otimes s_{\beta} \otimes s_{\gamma}\right)|\chi\rangle\right)\right|^{2}} \\
= & {\left[\sum _ { \alpha = 1 } ^ { N _ { 1 } } \sum _ { \beta = 1 } ^ { N _ { 2 } } \sum _ { \gamma = 1 } ^ { N _ { 3 } } \sum _ { \delta = 1 } ^ { 9 } \left(\left\langle\chi^{*}\left|S_{\alpha \beta \gamma}^{T} s^{\delta} S_{\alpha \beta \gamma}\right| \chi\right\rangle\right.\right.} \\
\times \quad & \left.\left.\left\langle\chi\left|S_{\alpha \beta \gamma}^{T} s^{\delta} S_{\alpha \beta \gamma}\right| \chi^{*}\right\rangle\right)\right]^{1 / 2}
\end{aligned}
$$

where $S_{\alpha \beta \gamma}=s_{\alpha} \otimes s_{\beta} \otimes s_{\gamma}, s^{\delta}$ are defined by eqs. (2-4), and the superscript $T$ denotes transposition operation.

\section{FULL SEPARABILITY CRITERION FOR MIXED STATES}

On the basis of $\mathcal{C}(|\chi\rangle)$ for pure states, the corresponding quantity $\mathcal{C}(\rho)$ for mixed states $\rho$ defined in $C_{d \times d}\left(d=n_{1} \times n_{2} \times n_{3}\right)$ is then given as the convex of

$$
\mathcal{C}(\rho)=\inf \sum_{i} p_{i} \mathcal{C}\left(\left|\Psi_{i}\right\rangle\right)
$$

of all possible decompositions into pure states $\left|\Psi_{i}\right\rangle$ with

$$
\rho=\sum_{i} p_{i}\left|\Psi_{i}\right\rangle\left\langle\Psi_{i}\right|, p_{i} \geq 0
$$

$\mathcal{C}(\rho)$ vanishes if and only if $\rho$ is fully separable. Substitute eq. (7) into eq. (8), one can get

$\mathcal{C}(\rho)=\inf _{U} \sum_{i} p_{i}\left[\sum_{\alpha=1}^{N_{1}} \sum_{\beta=1}^{N_{2}} \sum_{\gamma=1}^{N_{3}} \sum_{\delta=1}^{9} \mid\left. C^{\delta}\left(S_{\alpha \beta \gamma}\left|\Psi_{i}\right\rangle\right)\right|^{2}\right]^{1 / 2}$.

It is obvious that if the infimum of eq. (10) can be provided, one can obtain a sufficient and necessary condition of separability for mixed states. However, it seems to be impossible for higher-dimensional systems. One can only give a lower bound as a necessary condition. Therefore, a lower bound with strong sufficiency or convenient for calculations is expected.

According to the matrix notation [7] of equation (9), one can obtain $\rho=\Psi W \Psi^{\dagger}$, where $W$ is a diagonal matrix with $W_{i i}=p_{i}$, the columns of the matrix $\Psi$ correspond to the vectors $\left|\Psi_{i}\right\rangle$. Due to the eigenvalue decomposition: $\rho=\Phi M \Phi^{\dagger}$, where $M$ is a diagonal matrix whose diagonal elements are the eigenvalues of $\rho$, and $\Phi$ is a unitary matrix whose columns are the eigenvectors of $\rho$, one can 
obtain $\Psi W^{1 / 2}=\Phi M^{1 / 2} U$, where $U \in C^{r^{\prime} \times N}$ is a Rightunitary matrix, with $N$ and $r^{\prime}$ being the column number of $\Psi$ and the rank of $\rho$. Therefore, based on the matrix notation, eq. (10) can be rewritten as

$$
\begin{aligned}
\mathcal{C}(\rho) \geqslant & \inf _{U} \sqrt{\sum_{\alpha, \beta, \gamma, \delta}\left|U^{T} M^{1 / 2} \Phi^{T} S_{\alpha \beta \gamma}^{T} s^{\delta} S_{\alpha \beta \gamma} \Phi M^{1 / 2} U\right|_{i i}^{2}} \\
= & \inf _{U}\left\{\sum _ { \alpha , \beta , \gamma , \delta } \left[\left(U^{T} M^{1 / 2} \Phi^{T} S_{\alpha \beta \gamma}^{T} s^{\delta} S_{\alpha \beta \gamma} \Phi M^{1 / 2} U\right)\right.\right. \\
& \left.\left.\times\left(U^{\dagger} M^{1 / 2} \Phi^{\dagger} S_{\alpha \beta \gamma}^{T} s^{\delta} S_{\alpha \beta \gamma} \Phi^{*} M^{1 / 2} U^{*}\right)\right]_{i i}\right\}^{1 / 2}(11)
\end{aligned}
$$

where the Minkowski inequality

$$
\sum_{m} p_{m} \sqrt{\sum_{n} x_{m n}^{2}} \geqslant \sqrt{\sum_{n}\left(\sum_{m} p_{m} x_{m n}\right)^{2}}
$$

is used. According to Ref. [7], one can directly obtain a lower bound of $\mathcal{C}(\rho)$ as $\max _{\mathbf{z}} \tilde{\lambda}_{1}(\mathbf{z})-$ $\sum_{i>1} \tilde{\lambda}_{i}(\mathbf{z})$, where $\tilde{\lambda}_{j}(\mathbf{z})$ are the singular values of $\sum_{j=1}^{I} z_{j} M^{1 / 2} \Phi^{T} S_{\alpha \beta \gamma}^{T} s^{\delta} S_{\alpha \beta \gamma} \Phi M^{1 / 2}$ in decreasing order with $\mathbf{z}=\left[z_{1}, z_{2}, \cdots, z_{I}\right]$ a group of optimal complex parameters. It can be easily found that the number of optimal parameters $\left(I=9 N_{1} \cdot N_{2} \cdot N_{3}\right)$ is too large to be conveniently used to calculations for higher-dimensional systems yet. However, it will be found that by kronecker product approximation technique, not only might the number of optimal parameters be further reduced, but also one can calculate the lower bound in different approximation degrees. In particular, we can provide an analytic approximation for weakly mixed states.

In fact, if replacing " $\times$ " of eq. (11) by " $\otimes "$, eq. (11) can be rewritten as

$$
\begin{aligned}
\mathcal{C}(\rho) \geq & \inf _{U}\left\{\sum _ { \alpha , \beta , \gamma , \delta } \left[\left(U^{T} M^{1 / 2} \Phi^{T} S_{\alpha \beta \gamma}^{T} s^{\delta} S_{\alpha \beta \gamma} \Phi M^{1 / 2} U\right)\right.\right. \\
& \left.\left.\otimes\left(U^{\dagger} M^{1 / 2} \Phi^{\dagger} S_{\alpha \beta \gamma}^{T} s^{\delta} S_{\alpha \beta \gamma} \Phi^{*} M^{1 / 2} U^{*}\right)\right]_{i i}^{i i}\right\}^{1 / 2} \\
= & \inf _{U}\left\{\left[\left(U^{T} \otimes U^{\dagger}\right) A\left(U \otimes U^{*}\right)\right]_{i i}^{i i}\right\}^{1 / 2}
\end{aligned}
$$

where

$$
A=\sum_{\alpha, \beta, \gamma} \sum_{\delta=1}^{9}\left(\rho^{1 / 2}\right)^{T} \mathbf{S}_{\alpha \beta \gamma}^{T} \Sigma^{\delta} \mathbf{S}_{\alpha \beta \gamma}\left(\rho^{1 / 2}\right),
$$

defined in $C_{d \times d} \otimes C_{d \times d}$, and $\rho^{1 / 2}=\left(\Phi M^{1 / 2}\right) \otimes\left(\Phi M^{1 / 2}\right)^{*}$, $\Sigma^{\delta}=s^{\delta} \otimes s^{\delta}, \mathbf{S}_{\alpha \beta \gamma}=S_{\alpha \beta \gamma} \otimes S_{\alpha \beta \gamma}$. The other indices in above equation are all defined the same as previous sections. Even though the value of eq. (11) is not changed, the implied meaning is quite different, which means that we have copied the given quantum state in a conjugate Hilbert space and we consider the separability of the state in a doubled Hilbert space. The distinct advantage is that eq. (12) allows us to employ the kronecker product approximation technique $[16,17]$.
Next we will employ the kronecker product approximation technique on $A$ to derive a lower bound of eq. (12). Based on the technique, $A$ should be converted [19] into $\tilde{A}$ by

$$
\tilde{A}=V_{12}\left(A V_{12}\right)^{T_{2}},
$$

where the superscript $T_{2}$ denotes partial transposition on the second subspace [18], $V_{12}$ is swap operator [19] defined as

$$
\begin{aligned}
V_{12} & =\sum_{i k j^{\prime} k^{\prime}} \delta_{j k^{\prime}} \delta_{j^{\prime} k}|j\rangle\left\langle j^{\prime}|\otimes| k\right\rangle\left\langle k^{\prime}\right|, \\
j, k^{\prime} & =1, \cdots, d, j^{\prime}, k=1, \cdots, d .
\end{aligned}
$$

$\tilde{A}$ has the singular value decomposition:

$$
\tilde{A}=U \Sigma V^{\dagger}=\sum_{i=1}^{r} \sigma_{i} u_{i} v_{i}^{\dagger}
$$

where $u_{i}, v_{i}$ are the $i$ th columns of the unitary matrices $U$ and $V$, respectively; $\Sigma$ is a diagonal matrix with elements $\sigma_{i}$ decreasing for $i=1, \cdots, r ; r$ is the rank of $\tilde{A}$. Thus, based on Ref. [16,17] $A$ can always be written by

$$
A=\sum_{i}^{r} \mathcal{A}_{i} \otimes \mathcal{B}_{i}=\sum_{i}^{r} \sigma_{i} \mathcal{A}_{i}^{\prime} \otimes \mathcal{B}_{i}^{\prime},
$$

where $\operatorname{Vec}\left(\mathcal{A}_{i}\right)=\sqrt{\sigma_{i}} u_{i}$ and $\operatorname{Vec}\left(\mathcal{B}_{i}\right)=\sqrt{\sigma_{i}} v_{i}^{*}$. For any a $p \times q$ matrix $M=\left[m_{i j}\right]$ with entries $m_{i j}[20], V e c(M)$ is defined by

$V e c(M)=\left[m_{11}, \cdots, m_{p 1}, m_{12}, \cdots, m_{p 2}, \cdots, m_{1 q}, \cdots, m_{p q}\right]^{T}$.

One can find from eq. (13) that if the two subspace that $A$ is defined in is exchanged, $A$ will converted into $A^{*}$, hence one has $\mathcal{B}_{i}=\mathcal{A}_{i}^{*}$, i.e.

$$
A=\sum_{i}^{r} \mathcal{A}_{i} \otimes \mathcal{A}_{i}^{*}=\sum_{i}^{r} \sigma_{i} \mathcal{A}_{i}^{\prime} \otimes \mathcal{A}_{i}^{\prime *}
$$

Substitute eq. (16) into eq. (12), eq. (12) can be given by

$$
\mathcal{C}(\rho) \geq \inf _{U} \sum_{i}^{N} \sum_{j=1}^{r}\left|\left(U^{T} \mathcal{A}_{j} U\right)_{i i}\right|^{2}
$$

The infimum can be employed to test the full separability of $\rho$.

In terms of the Cauchy-Schwarz inequality $\left(\sum_{i} x_{i}^{2}\right)^{1 / 2} \times\left(\sum_{i} y_{i}^{2}\right)^{1 / 2} \geqslant \sum_{i} x_{i} y_{i}$ and $\sum_{i}\left|x_{i}\right| \geq\left|\sum_{i} x_{i}\right|, \mathcal{C}(\rho)$ given by eq. (16) can arrive at

$$
\mathcal{C}(\rho) \geq \inf _{U} \sum_{i}^{N}\left|U^{T}\left(\sum_{j=1}^{r} z_{j} \mathcal{A}_{j}\right) U\right|_{i i}
$$


where $z_{j}=x_{j} \exp \left(i \phi_{j}\right)$, with $x_{j} \geq 0, \sum_{j} x_{j}^{2}=$ 1.Therefore the infimum of eq. (18) can be given by $\max \lambda_{1}(\mathbf{z})-\sum_{i>1} \lambda_{i}(\mathbf{z})$, where $\lambda_{j}(\mathbf{z})$ are the singular values of $\left(\sum_{j=1}^{r} z_{j} \mathcal{A}_{j}\right)$ in decreasing order [7], with $\mathbf{z}=\left[z_{1}, z_{2}, \cdots, z_{r}\right]$. Note that $r \leq d^{2}$ is usually much smaller than $d^{2}$ in practical calculations. In particular, one can consider different numbers of $\sigma_{j}$ in decreasing order and correspondingly introduce optimal parameters, which will might provide approximate lower bounds in different degrees. In this sense, the number of optimal parameters can be dramatically reduced. In fact, it is very possible that $\mathcal{A}_{j}$ corresponding to the maximal $\sigma_{j}$ can give the main contribution [8] to the infimum of eq. (18). That is to say the lower bound of $\mathcal{C}(\rho)$ can be given by $\lambda_{1}-\sum_{i>1} \lambda_{i}$ with $\lambda_{j}$ the singular values of $\mathcal{A}_{j}$, which is an analytic approximation.

For weakly mixed states i.e. quasi pure states, an analytic approximation of $\mathcal{C}(\rho)$ can also be introduced [21]. According to eq. (13) and kronecker approximation technique, $A$ can also be given in the following way

$$
\begin{gathered}
A_{l^{\prime} m^{\prime}}^{l m}=\sum_{\alpha, \beta, \gamma} \sum_{\delta=1}^{9} \sqrt{u_{l} u_{l^{\prime}} u_{m} u_{m^{\prime}}} \\
\times\left(\left\langle\Psi_{l}^{*}\left|S_{\alpha \beta \gamma}^{T} s^{\delta} S_{\alpha \beta \gamma}\right| \Psi_{l^{\prime}}\right\rangle \times\left\langle\Psi_{m}\left|S_{\alpha \beta \gamma}^{T} s^{\delta} S_{\alpha \beta \gamma}\right| \Psi_{m^{\prime}}^{*}\right\rangle\right),
\end{gathered}
$$

where $\Psi_{\alpha}$ and $u_{\alpha}$ denote the $\alpha$ th eigenvector and eigenvalue, and all the other quantities are defined similar to those in eq. (7). According to the symmetry of $A$ given by eq. (16) and the kronecker product approximation technique in above section, $A$ can be formally written as

$$
A_{l^{\prime} m^{\prime}}^{l m}=\sum_{\alpha} T_{l m}^{\alpha}\left(T_{l^{\prime} m^{\prime}}^{\alpha}\right)^{*}
$$

The density matrix of quasi pure states has one single eigenvalue $\mu_{1}$ that is much larger than all the others, which induces a natural order in terms of the small eigenvalues $\mu_{i}, i>1$. Due to the same reasons to those in Ref. [21], here we consider the second order elements of type $A_{11}^{l m}$. Therefore, one can have the approximation

$$
A_{l^{\prime} m^{\prime}}^{l m} \simeq \tau_{l m} \tau_{l^{\prime} m^{\prime}}^{*} \text { with } \tau_{l m}=\frac{A_{11}^{l m}}{\sqrt{A_{11}^{11}}}
$$

In this sense, eq. (18) can be simplified significantly:

$$
\mathcal{C}(\rho) \simeq \mathcal{C}_{a}(\rho)=\inf _{U} \sum_{i}\left|U^{T} \tau U\right|_{i i}
$$

$\mathcal{C}_{a}(\rho)$ can be given by

$$
\mathcal{C}_{a}(\rho)=\max \left\{\lambda_{1}-\sum_{i>1} \lambda_{i}, 0\right\}
$$

where $\lambda_{i}$ is the singular value of $\tau$ in decreasing order.
Consider two $(2 \times 2 \times 3)$-dimensional quasi pure states constructed respectively by

$$
\rho_{1}(x)=x\left|G H Z^{\prime}\right\rangle\left\langle G H Z^{\prime}\right|+(1-x) \mathbf{1}_{12}
$$

and

$$
\rho_{2}(x)=x\left|W^{\prime}\right\rangle\left\langle W^{\prime}\right|+(1-x) \mathbf{1}_{12},
$$

where

$$
\left|G H Z^{\prime}\right\rangle=\frac{1}{2}(|000\rangle+|101\rangle+|011\rangle+|112\rangle),
$$

and

$$
\left|W^{\prime}\right\rangle=\frac{1}{\sqrt{3}}(|000\rangle+|011\rangle+|112\rangle) .
$$

Note that $\left|G H Z^{\prime}\right\rangle$ and $\left|W^{\prime}\right\rangle$ given in Ref. [11] correspond to $G H Z$ class and $W$ class with high local rank, respectively. The two states can be considered as quasi pure states for $x \geq 0.3$. By the calculation, one can find that $\mathcal{C}_{a}\left(\rho_{1}\right)$ and $\mathcal{C}_{a}\left(\rho_{2}\right)$ are both nonzero. What is more, for the quasi pure states generated by the mixture of maximally mixed state (identity matrix) and tripartite GHZ state in $3 \times 3 \times 3$ dimension, the corresponding $\mathcal{C}_{a}(\rho)$ s can all be shown to be nonzero for $x \geq 0.3$. We also study some $(2 \times 2 \times 3)$-dimensional quasi pure states $\rho$ by the mixture of maximally mixed state and random semiseparable pure states generated by Matlab, numerical results show that $\mathcal{C}_{a}(\rho)$ are nonzero if $\rho$ are strict quasi pure states. All above show the sufficiency of our criterion for testing the entanglement of high-dimensional mixed systems.

\section{CONCLUSION AND DISCUSSION}

In summary, we have utilized an intuitive approach to generalize the criterion to high-dimensional tripartite systems. The generalized criterion for pure states can be conveniently extended to the case of mixed states by utilizing the kronecker product approximate technique. The lower bound for mixed states can provide necessary conditions to test the full separability. Compared with the previous criteria, the criterion introduced here can effectively reduce the restrictive conditions. However, the criterion is not an entanglement monotone. Numerical results show that our criterion for high-dimensional systems is even sufficient condition of full separability for strict quasi pure states.

\section{ACKNOWLEDGEMENT}

This work was supported by the National Natural Science Foundation of China, under Grant Nos. 10575017 and 60472017 . 
[1] M. A. Nielsen and I. L. Chuang, Quantum Computation and Quantum Information (Cambridge University Press, Cambridge, 2000).

[2] M. Zukowski, A. Zeilinger, M. A. Horne, and A. K. Ekert, Phys. Rev. Lett. 71, 4287 (1993).

[3] C. H. Bennett, et al., Phys. Rev. Lett.70,1895 (1993).

[4] C. H. Bennett and S. Wiesner, Phys. Rev. Lett. 69, 2881 (1992).

[5] W. K. Wootters, Phys. Rev. Lett. 80, 2245 (1998).

[6] A.Uhlmann, Phys. Rev. A 62, 032307 (2000).

[7] K. Audenaert, F.Verstraete and De Moor, Phys. Rev. A 64, 052304 (2001).

[8] Florian Mintert, Marek Kuś, and Andreas Buchleitner, Phys. Rev. Lett. 92, 167902 (2004).

[9] Valerie Coffman, Joydip Kundu, and William K. Wootters, Phys. Rev. A 61, 052306 (2000).

[10] W. Dür, G. Vidal, and J. I. Cirac, Phys. Rev. A 62, 062314 (2000).

[11] A. Miyake, Phys. Rev. A 67, 012108 (2003).

[12] Andreas Osterloh, Jens Siewert, Phys. Rev. A 72, 012337
(2005).

[13] Chang-shui Yu, He-shan Song, Phys. Rev. A 73, 022325 (2006).

[14] A. R. R. Carvalho, F. Mintert, A. Buchleitner, Phys. Rev. Lett. 93, 230501 (2004);

[15] Chang-shui Yu, He-shan Song, Phys. Rev. A 72, 022333 (2005).

[16] C. F. Van Loan and N. P. Pitsianis, in Linear Algebra for Large Scale and Real Time Applications, edited by M. S. Moonen and G. H. Golub (Kluwer, Dordrecht, 1993), pp. 293-314.

[17] N. P. Pitsianis, Ph.D. thesis, Cornell University, New York, 1997.

[18] A. Peres, Phys. Rev. Lett. 76, 1413 (1996).

[19] Heng Fan, e-print quant-ph/0210168.

[20] R. A. Horn and C. R. Johnson, Matrix Analysis (Cambridge University Press, New York, 1985).

[21] Florian Mintert, André R. R. Carvalho, Marek Kuś, and Andreas Buchleitner, Physics Report 415, 207 (2005). 